\title{
Flow of a Newtonian fluid in a non-uniform wavy and permeable tube
}

\author{
Tesfahun Berhane \\ Bahir Dar University, Department of Mathematics, Bahir Dar, Ethiopia
}

Received: 8 November 2016, Accepted: 19 July 2017

Published online: 18 October 2017.

\begin{abstract}
In this paper, a viscous incompressible fluid flow in a wavy non-uniform rigid tube with permeable wall by taking in to account the influence of slip velocity at the wall is studied. It is assumed that the exchange of fluid across the wall obeys Starling's hypothesis, that is, the rate of flow per unit area through the wall surface is proportional to the difference between the pressure of the fluid within and outside the wall. The nonlinear governing equations of motion are linearized by perturbation method by assuming $\delta$ (ratio of inlet width to wavelength) as a small parameter and the resulting equations are solved by numerical methods. The effects of permeability parameter $(\alpha)$, slope parameter $(k)$, slip coefficient $(\xi)$ and Reynolds number $\left(R_{e}\right)$ on the velocity profiles, pressure and flow rate are presented graphically. Results concerning the velocity, pressure and flow rate, indicate that the slip and permeability parameters influence the flow field significantly. Discussions are made from physiological point of view.
\end{abstract}

Keywords: Fluid flow, non-uniform tube.

\section{Introduction}

For several decades there has been an effort to understand the mechanism of kidney in terms of mathematical models. Kidney, one of the excretory organs in human body, perform two major functions, they excrete end products of body metabolism and control concentrations of most of the constitutes of body fluids. The basic functional unit of kidney is nephron. Each kidney contains over a million tiny units of nephrons, all similar in structure and function. Each nephron functions independently and in most instances it is sufficient to study the function of nephron to understand the mechanism of kidney in terms of mathematical models.

In nephrons, the portion after the Bowman capsule is called proximal convoluted tubule. It is the place where most of the wanted substances, like water, glucose and electrolytes are reabsorbed back into the plasma and unwanted substances pass into urine. The proximal renal tubules are permeable as it is the place where most of the reabsorption is taking place. It is therefore suitable to consider a mathematical model for renal flow with non-uniform tube of varying cross-section with slip velocity at the wall.

Study of viscous fluid flow in tubes of varying cross section with permeable wall is significant because of its application to both physiological and engineering flow problems. The flow of fluid in a renal tubule has been studied by different authors. Macey [2] formulated the problem as the flow of an incompressible viscous fluid through a circular tube with linear rate of reabsorption at the wall. Whereas, Kelman [1] found that the bulk flow in the proximal tubule decays exponentially with the axial distance. Then, Macey [3] used this condition to solve the equations of motion and 
mentioned that the longitudinal velocity profile is parabolic and the drop in mean pressure is proportional to the mean axial flow. Marshall and Trowbridge [4] and Palatt et.al [5] used physical conditions existing at the rigid permeable tube instead of prescribing the flux at the wall as a function of axial distance.

In all the above studies researchers considered the renal tubule as cylindrical tube of uniform cross-section, while in general such tubles may not have uniform cross-section throughout their length. Radhakrishnamacharya et al [6] made an attempt to understand the flow through the renal tubule by studying the hydrodynamical aspects of an incompressible viscous fluid in a circular tube of varying cross-section with reabsorption at the wall. Chandra and Prasad [7] analyzed flow in a rigid tube of slowly varying cross-section with absorbing wall by considering the exchange of fluid across the boundary obey's Starling's hypothesis. Chaturani and Ranganatha [8] considered fluid flow through a diverging/converging tube with variable wall permeability. Recently, Muthu and Tesfahun [13] studied the effects of slope parameter and reabsorption coefficient on the flow of fluid in a symmetric channel with varying cross section.

In all the above studies the boundary condition at the wall is taken as no-slip condition. The no-slip boundary condition is one of the cornerstones on which the mechanics of the viscous liquids is built. However, there are situations where this assumption does not hold [11]. The effect of slip at the wall is quite significant as illustrated by Elshahed [12]. So that we need to study the effect of slip parameter on a flow in renal tubules.

On the other hand, various authors studied a blood flow in a capillary under Starling hypothesis by considering the blood vessel as a straight tube. Oka and Murata [14] studied a hydrodynamical theory of the steady slow motion of blood through a capillary with permeable wall, where it is assumed that the exchange of fluid across the capillary wall obeys Starling's hypothesis, that is, the rate of flow per unit area through the wall surface is proportional to the difference between the pressure of the fluid within and outside of the capillary.

Mariamma and Majhi [15] extended Okas's model to non-linear case and considered the steady laminar flow of blood as a homogeneous Newtonian fluid in tube with permeable wall. Elshahed [12] extended the work of Mariamma et al by taking the influence of slip velocity at the membrane surface. However, straight tube assumption is an idealization. Hence, in this analysis we study the renal flow under Starling hypothesis for non-uniform nature of the proximal tubule with slip velocity at the wall.

The objective of the present paper is to understand the flow through renal tubule by studying the hydrodynamical aspect of an incompressible viscous fluid in a rigid tube of varying cross-section with reabsorption at the wall by considering a slip velocity at the wall of the tube. The fluid exchange across the wall obeys Starling hypothesis. That is, the rate of flow per unit area through the wall surface is proportional to the difference between the pressure of the fluid within and outside the wall. The boundary of the tube wall varies with $x$. It is taken as

$$
\eta(x)=d+k_{1} x+a \sin \left(\frac{2 \pi x}{\lambda}\right)
$$

where $d$ is the radius of the tube at the inlet (at $x=0), k_{1}$ is a constant whose magnitude depends on the length of the tube exit and inlet dimensions and which is assumed to be $\ll 1, a$ is the amplitude and $\lambda$ is the wave length, it is shown in figure 1 below. 


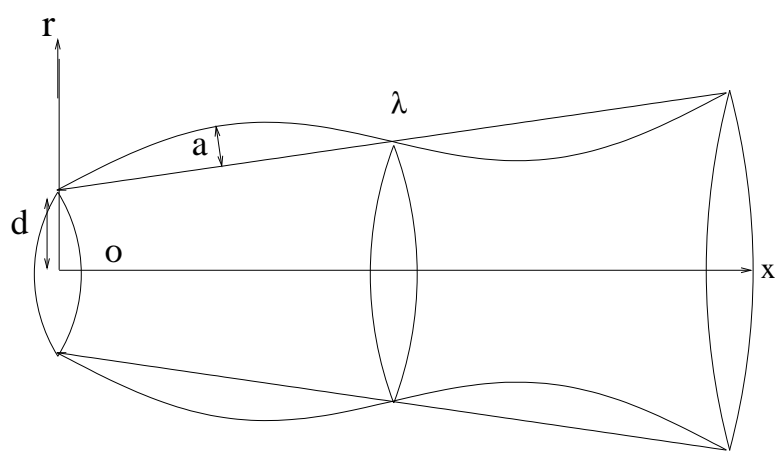

Fig. 1: Geometry of 3 dimensional renal tubule.

\section{Mathematical formulation of the problem}

An incompressible fluid flow through a tube with slowly varying cross-section is considered and it is given by equation (1). The tube is long enough to neglect the initial and end effects. The motion of the fluid is assumed to be laminar and steady. The governing equations of such fluid motion are given by

$$
\begin{aligned}
& \frac{1}{r} \frac{\partial}{\partial r}(r v)+\frac{\partial u}{\partial x}=0 \\
& u \frac{\partial u}{\partial x}+v \frac{\partial u}{\partial r}=-\frac{1}{\rho} \frac{\partial p}{\partial x}+v\left(\frac{\partial^{2} u}{\partial x^{2}}+\frac{\partial^{2} u}{\partial r^{2}}+\frac{1}{r} \frac{\partial u}{\partial r}\right) \\
& u \frac{\partial v}{\partial x}+v \frac{\partial v}{\partial r}=-\frac{1}{\rho} \frac{\partial p}{\partial r}+v\left(\frac{\partial^{2} v}{\partial x^{2}}+\frac{\partial^{2} v}{\partial r^{2}}-\frac{v}{r^{2}}+\frac{1}{r} \frac{\partial v}{\partial r}\right)
\end{aligned}
$$

where $u$ and $v$ are the velocity components along the $x$ and $r$ axes respectively, $p$ is the pressure, $\rho$ density of the fluid and $v=\frac{\mu}{\rho}$ is kinematic viscosity. In this case the boundary conditions are

(a) The regularity condition requires

$$
v=0 \quad \text { and } \quad \frac{\partial u}{\partial r}=0 \text { at } r=0 .
$$

(b) The tangential velocity at the wall is not zero (slip condition). That is,

$$
\mathbf{v} \cdot \hat{t_{w}}=-\frac{\sqrt{\gamma}}{\beta} \frac{\partial}{\partial r}\left(\mathbf{v} \cdot \hat{t_{w}}\right) \quad \text { at } \quad r=\eta(x) .
$$

where $\beta$ is slip parameter, $\gamma$ is the specific permeability of the porous medium, $\hat{t_{w}}$ is the unit tangent vector at the wall and $\mathbf{v}$ is velocity vector.

(c) Fluid exchange across the wall is given by Starling's law. That is,

$$
\mathbf{v} \cdot \hat{n_{w}}=\alpha\left(p-p_{\text {ext }}\right) \quad \text { at } \quad r=\eta(x)
$$

where $\hat{n_{w}}$ is the unit normal at the wall, $\alpha$ is the measure of permeability of the wall and ( $\left.p_{\text {ext }}\right)$ is net external pressure acting on the wall which is taken to be a constant.

(d) Further, we specify the average pressure at the two end cross-sections of the tube as

$$
\bar{p}=p_{\text {in }} \quad \text { at } \quad x=0 \quad \text { and } \quad \bar{p}=p_{\text {out }} \quad \text { at } \quad x=\lambda .
$$


To non-dimensionalize the governing equation we use the following non-dimensional quantities

$$
\begin{aligned}
& x^{\prime}=\frac{x}{\lambda}, \\
& r^{\prime}=\frac{r}{d}, \\
& \eta^{\prime}=\frac{\eta}{d}, \\
& u^{\prime}=\frac{2 \pi d^{2} u}{Q_{0}} \\
& v^{\prime}=\frac{2 \pi d \lambda v}{Q_{0}}, \\
& \alpha^{\prime}=\frac{\alpha \mu \lambda^{2}}{d^{3}}, \\
& p^{\prime}=\frac{2 \pi d^{4} p}{\lambda \mu Q_{0}}, \\
& p_{\text {ext }}^{\prime}=\frac{2 \pi d^{4} p_{\text {ext }}}{\lambda \mu Q_{0}}
\end{aligned}
$$

where $Q_{0}$ is the flux across the cross-section at $x=0$, equations (2) - (4) are transformed to the non-dimensional form as (after dropping the primes)

$$
\begin{aligned}
& \frac{1}{r} \frac{\partial}{\partial y}(r v)+\frac{\partial u}{\partial x}=0 \\
& R_{e} \delta\left[u \frac{\partial u}{\partial x}+v \frac{\partial u}{\partial r}\right]=-\frac{\partial p}{\partial x}+\delta^{2} \frac{\partial^{2} u}{\partial x^{2}}+\frac{\partial^{2} u}{\partial r^{2}}+\frac{1}{r} \frac{\partial u}{\partial r} \\
& R_{e} \delta^{3}\left[u \frac{\partial v}{\partial x}+v \frac{\partial v}{\partial r}\right]=-\frac{\partial p}{\partial r}+\delta^{2}\left[\delta^{2} \frac{\partial^{2} v}{\partial x^{2}}+\frac{\partial^{2} v}{\partial r^{2}}-\frac{v}{r^{2}}+\frac{1}{r} \frac{\partial v}{\partial r}\right]
\end{aligned}
$$

where $\delta=\frac{d}{\lambda}$ and $R_{e}=\frac{Q_{0}}{2 \pi d v}$. And the boundary conditions (5) - (8) become

$$
\begin{aligned}
& v=0 \quad \text { and } \quad \frac{\partial u}{\partial r}=0 \text { at } r=0 \\
& u+\delta^{2} \frac{d \eta}{d x} v=-\xi\left(\frac{\partial u}{\partial r}+\delta^{2} \frac{d \eta}{d x} \frac{\partial v}{\partial r}\right) \text { at } r=\eta(x)=1+k x+\varepsilon \sin (2 \pi x) \\
& v-\frac{d \eta}{d x} u=\alpha\left(p-p_{\text {ext }}\right)\left(1+\delta^{2}\left(\frac{d \eta}{d x}\right)^{2}\right)^{\frac{1}{2}} \text { at } r=\eta(x)=1+k x+\varepsilon \sin (2 \pi x) \\
& \bar{p}=p_{\text {in }} \text { at } x=0 \text { and } \bar{p}=p_{\text {out }} \text { at } x=1 .
\end{aligned}
$$

where $\varepsilon=\frac{a}{d}, k=\frac{k_{1} \lambda}{d}$ and $\xi=\frac{\sqrt{\gamma}}{\beta d}$.

The parameter $R_{e}$ is the Reynolds number and $\delta$ is the wave-number (the ratio of inlet width to the wavelength). $\varepsilon$ is amplitude ratio (the ratio of amplitude to the inlet width), $k$ is slope parameter and $\xi$ is the slip coefficient.

\section{Solving the governing equations}

In the present analysis, assuming the wave number $\delta \ll 1$ (long wavelength approximation), we shall seek a solution for equations (9) - (15) in the form of a power series in terms of $\delta$ as

$$
(u, v, p)=\left(u_{0}, v_{0}, p_{0}\right)+\delta\left(u_{1}, v_{1}, p_{1}\right)+\ldots
$$

Substituting equation (16) in equations (9) - (15) and collecting coefficients of various like powers of $\delta$, we get the following sets of equations.

\section{Zeros order case.}

$$
\frac{1}{r} \frac{\partial}{\partial r}\left(r v_{0}\right)+\frac{\partial u_{0}}{\partial x}=0, \quad \frac{\partial^{2} u_{0}}{\partial r^{2}}+\frac{1}{r} \frac{\partial u_{0}}{\partial r}=\frac{\partial p_{0}}{\partial x}, \quad \frac{\partial p_{0}}{\partial r}=0
$$

The boundary conditions are

$$
v_{0}=0 \quad \text { and } \quad \frac{\partial u_{0}}{\partial r}=0 \quad \text { at } \quad r=0
$$




$$
\begin{gathered}
u_{0}=-\xi \frac{\partial u_{0}}{\partial r} \quad \text { and } \quad v_{0}-\frac{d \eta}{d x} u_{0}=\alpha\left(p_{0}-p_{\text {ext }}\right) \quad \text { at } \quad r=\eta(x) \\
\overline{p_{0}}=p_{\text {in }} \quad \text { at } \quad x=0 \quad \text { and } \quad \overline{p_{0}}=p_{\text {out }} \quad \text { at } \quad x=1 .
\end{gathered}
$$

\section{First order case.}

$$
\frac{1}{r} \frac{\partial}{\partial r}\left(r v_{1}\right)+\frac{\partial u_{1}}{\partial x}=0, \quad \frac{\partial^{2} u_{1}}{\partial r^{2}}+\frac{1}{r} \frac{\partial u_{1}}{\partial r}=\frac{\partial p_{1}}{\partial x}+R_{e}\left[u_{0} \frac{\partial u_{0}}{\partial x}+v_{0} \frac{\partial u_{0}}{\partial r}\right], \quad \frac{\partial p_{1}}{\partial r}=0
$$

The boundary conditions are

$$
\begin{gathered}
v_{1}=0 \quad \text { and } \quad \frac{\partial u_{1}}{\partial r}=0 \quad \text { at } \quad r=0 \\
u_{1}=-\xi \frac{\partial u_{1}}{\partial r} \quad \text { and } \quad v_{1}-\frac{d \eta}{d x} u_{1}=\alpha p_{1} \quad \text { at } \quad r=\eta(x) \\
\overline{p_{1}}=0 \quad \text { at } \quad x=0 \text { and } \overline{p_{1}}=0 \text { at } x=1 .
\end{gathered}
$$

Here we can proceed to higher orders of $\delta$. However, since we are looking for an approximate analytical solution for the problem, we consider up to first order of equations. From equation (17) and the conditions (19) and (18), we get that

$$
u_{0}=\frac{d p_{0}}{d x}\left[\frac{1}{4}\left(r^{2}-\eta^{2}\right)-\frac{1}{2} \xi \eta\right]
$$

Integrating the equation of continuity (17) and using the boundary condition (18) along with the expression of $u_{0}$ from (25), we get

$$
v_{0}=-\frac{d^{2} p_{0}}{d x^{2}} \frac{r}{16}\left(r^{2}-2 \eta^{2}-4 \xi \eta\right)+\frac{d p_{0}}{d x} \frac{r}{4} \frac{d \eta}{d x}(\eta+\xi) .
$$

Further, using the condition (19) and the expression for $v_{0}$ from (26), we get the equation governing the zeroth order pressure $p_{0}$ as follows :

$$
\frac{d^{2} p_{0}}{d x^{2}}+\left(4 \frac{d \eta}{d x} \frac{\eta+3 \xi}{\eta^{2}+4 \xi \eta}\right) \frac{d p_{0}}{d x}=\frac{16 \alpha}{\eta^{3}+4 \xi \eta}\left(p_{0}-p_{\text {ext }}\right)
$$

Now, equation (27) with boundary condition (20) forms a two point boundary value problem, which is solved numerically.

Similarly, from equation (21) together with the boundary conditions (23) - (24) and with the help of equations (25) and (26), we get the expressions for $u_{1}$ and $v_{1}$ as follows :

$$
u_{1}=\frac{1}{4} \frac{d p_{1}}{d x}\left(r^{2}-A_{1}\right)+\frac{R_{e}}{2304} \frac{d p_{0}}{d x}\left[\frac{d^{2} p_{0}}{d x^{2}}\left(2 r^{6}+A_{2}-A_{3} r^{4}+A_{4} r^{2}-A_{5}-A_{6}\right)+\eta \frac{d \eta}{d x} \frac{d p_{0}}{d x}\left(A_{7} r^{2}-A_{8}-A_{9}\right)\right]
$$

where $A_{1}=\eta^{2}+2 \xi \eta, A_{2}=-2 \eta^{6}+9 \eta^{5}(\eta+2 \xi), A_{3}=9 \eta(\eta+2 \xi), A_{4}=36 \eta^{2}(\eta+2 \xi)^{2}, A_{5}=36 \eta^{4}(\eta+2 \xi)^{2}$, $A_{6}=\xi\left(12 \eta^{5}-36 \eta^{4}(\eta+2 \xi)+72 \eta^{3}(\eta+2 \xi)^{2}\right), A_{7}=72 \eta\left(\eta^{2}+3 \eta \xi+2 \xi^{2}\right), A_{8}=72 \eta^{3}\left(\eta^{2}+3 \eta \xi+2 \xi^{2}\right)$, $A_{9}=144 \xi \eta^{2}\left(\eta^{2}+3 \eta \xi+2 \xi^{2}\right)$.

$$
\begin{aligned}
v_{1} & =\frac{-r}{16}\left[\frac{d^{2} p_{1}}{d x^{2}}\left(r^{2}-2 A_{1}\right)-2 \frac{d A_{1}}{d x} \frac{d p_{1}}{d x}\right]-\frac{R_{e} r}{2304 \times 12}\left\{\left[\left(\frac{d^{2} p_{0}}{d x^{2}}\right)^{2}+\frac{d p_{0}}{d x} \frac{d^{3} p_{0}}{d x^{3}}\right]\left(3 r^{6}+6 A_{2}-2 A_{3} r^{4}+3 A_{4} r^{2}-6 A_{5}-6 A_{6}\right)\right. \\
& +\frac{d p_{0}}{d x} \frac{d^{2} p_{0}}{d x^{2}}\left(6 \frac{d A_{2}}{d x}-2 r^{4} \frac{d A_{3}}{d x}+3 r^{2} \frac{d A_{4}}{d x}-6 \frac{d A_{5}}{d x}-6 \frac{d A_{6}}{d x}\right)+3 \frac{d p_{0}}{d x}\left[2 \frac{d^{2} p_{0}}{d x^{2}} \frac{d \eta}{d x}+\frac{d p_{0}}{d x} \frac{d^{2} \eta}{d x^{2}}\right]\left(A_{7} r^{2}-2 A_{8}-2 A_{9}\right) \\
& \left.+3 \frac{d \eta}{d x}\left(\frac{d p_{0}}{d x}\right)^{2}\left[r^{2} \frac{d A_{7}}{d x}-2 \frac{d A_{8}}{d x}-2 \frac{d A_{9}}{d x}\right]\right\}
\end{aligned}
$$


and using the expression (29) and the boundary condition (23), we get the equation governing the first order pressure as :

$$
\begin{aligned}
\frac{d^{2} p_{1}}{d x^{2}} & -\frac{16}{\eta\left(\eta^{2}-2 A_{1}\right)}\left[\frac{\eta}{16} \frac{d A_{1}}{d x}-\frac{1}{4} \frac{d \eta}{d x}\left(\eta^{2}-A_{1}\right)\right] \frac{d p_{1}}{d x}+\frac{16 \alpha}{\eta\left(\eta^{2}-2 A_{1}\right)} p_{1} \\
& =\frac{-R_{e}}{144 \eta\left(\eta^{2}-2 A_{1}\right)}\left[\frac{\eta}{12}\left(B_{1}+B_{2}+B_{3}+B_{4}\right)+B_{5}+B_{6}\right]
\end{aligned}
$$

where

$$
\begin{aligned}
& B_{1}=\left(\left(\frac{d^{2} p_{0}}{d x^{2}}\right)^{2}+\frac{d p_{0}}{d x} \frac{d^{3} p_{0}}{d x^{3}}\right)\left(3 \eta^{6}+6 A_{2}-2 A_{3} \eta^{4}+3 A_{4} \eta^{2}-6 A_{5}-6 A_{6}\right) \\
& B_{2}=\frac{d p_{0}}{d x} \frac{d^{2} p_{0}}{d x^{2}}\left(6 \frac{d A_{2}}{d x}-2 \eta^{4} \frac{d A_{3}}{d x}+3 \eta^{2} \frac{d A_{4}}{d x}-6 \frac{d A_{5}}{d x}-6 \frac{d A_{6}}{d x}\right) \\
& B_{3}=\left(6 \frac{d p_{0}}{d x} \frac{d^{2} p_{0}}{d x^{2}} \frac{d \eta}{d x}+3\left(\frac{d p_{0}}{d x}\right)^{2} \frac{d^{2} \eta}{d x^{2}}\right)\left(A_{7} \eta^{2}-2 A_{8}-2 A_{9}\right) \\
& B_{4}=3\left(\frac{d P_{0}}{d x}\right)^{2} \frac{d \eta}{d x}\left(\eta^{2} \frac{d A_{7}}{d x}-2 \frac{d A_{8}}{d x}-2 \frac{d A_{9}}{d x}\right) \\
& B_{5}=\frac{d \eta}{d x} \frac{d p_{0}}{d x} \frac{d^{2} p_{0}}{d x^{2}}\left(2 \eta^{2}+A_{2}-A_{3} \eta^{4}+A_{4} \eta^{2}-A_{5}-A_{6}\right) \\
& B_{6}=\left(\frac{d p_{0}}{d x}\right)^{2}\left(\frac{d \eta}{d x}\right)^{2}\left(A_{7} \eta^{2}-A_{8}-A_{9}\right) .
\end{aligned}
$$

Hence, equation (30) with boundary condition (24) forms a two point boundary value problem, which is solved using numerical methods.

The non-dimensional volumetric flow rate $Q$ up to first order is given by

$$
\begin{aligned}
Q & =-\frac{\pi \eta^{3}}{8} \frac{d p_{0}}{d x}(\eta+\xi)+\pi \delta\left[\frac{1}{8} \frac{d p_{1}}{d x}\left(\eta^{4}-2 \eta^{2} A_{1}\right)+\frac{R_{e}}{1152} \frac{d p_{0}}{d x}\left\{\frac{\eta^{2}}{12} \frac{d^{2} p_{0}}{d x^{2}}\left(3 \eta^{6}+6 A_{2}-2 A_{3} \eta^{4}+3 A_{4} \eta^{2}-6 A_{5}-6 A_{6}\right)\right.\right. \\
& \left.\left.+\frac{\eta^{2}}{2} \frac{d p_{0}}{d x} \frac{d \eta}{d x}\left(A_{7} \eta^{2}-2 A_{8}-2 A_{9}\right)\right\}\right]
\end{aligned}
$$

\section{Results and discussion}

The main objective of this analysis is to study the behaviour of an incompressible fluid flow through a tube of converging/diverging and slowly varying cross-section with absorbing wall by considering slip velocity at the wall. It may be recalled that $k$ characterize the slope of the converging/diverging wavy wall. $k=0.1$ represents diverging tube, $k=0$ represents a normal (sinusoidal tube) and $k=-0.1$ represents a converging tube. $\varepsilon$ and $\alpha$ represent amplitude and permeability parameter of wavy wall, respectively.

The effects of these parameters on the radial velocity $v(x, r)$, pressure $(p)$ and flow rate $(Q)$ quantities are discussed. In all our numerical calculations, the following parameters are fixed as $\varepsilon=0.1$ and $\delta=0.1$. The values of $p_{\text {in }}$ and $p_{\text {out }}$ are taken as 15 and 5, respectively. In this analysis, we obtained numerical solution of equations (27) and (30) governing the zeroth and first order pressure values $\left(p_{0}\right.$ and $\left.p_{1}\right)$, and used these values to get the velocity profile and flow rate, consequently.

\subsection{Pressure $p$}

The pressure is calculated for different values of $k, R_{e}, \xi$ and $\alpha$. Fig. 2(a) displays the effect of slope parameter $k$ to pressure. We can notice that $p$ is less for the divergent tube than the normal or convergent tubes, and it is more for 
convergent tube than the normal/divergent tubes. This is because as the space for movement decreases, the velocity decreases which results in more pressure. As shown, in Fig. 2(b), when the permeability parameter $\alpha$ increases, the pressure decreases. This can justify the natural phenomenon that as most of the fluid goes out in a greater amount, the pressure is very less.

Fig. 3(a) shows the influence of Reynolds number $R e$ on $p$. As $R_{e}$ increases, pressure increases. The slip coefficient ( $\xi$ ) has a significant influence on the pressure as illustrated on Fig. 3(b). It can be observed that as the slip coefficient increases the pressure also increases hugely.

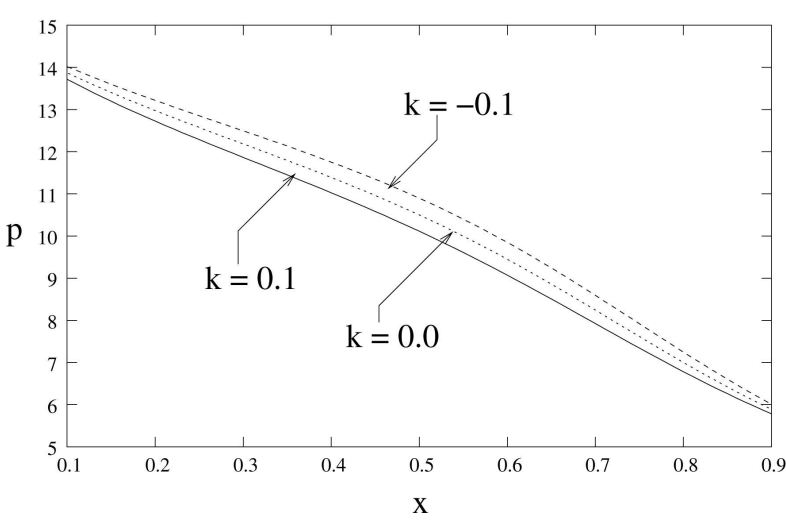

(a) Distribution of pressure $(p)$ with $x$. ( $R_{e}=0.05$, $\alpha=0.005, \xi=0.15$.

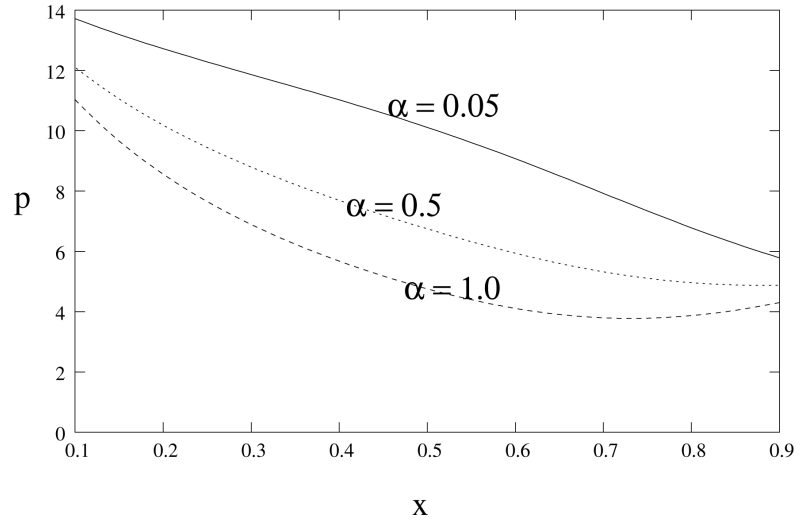

(b) Distribution of pressure $(p)$ with $x$. ( $R_{e}=0.05$, $\xi=0.15, k=0.1$.

Fig. 2

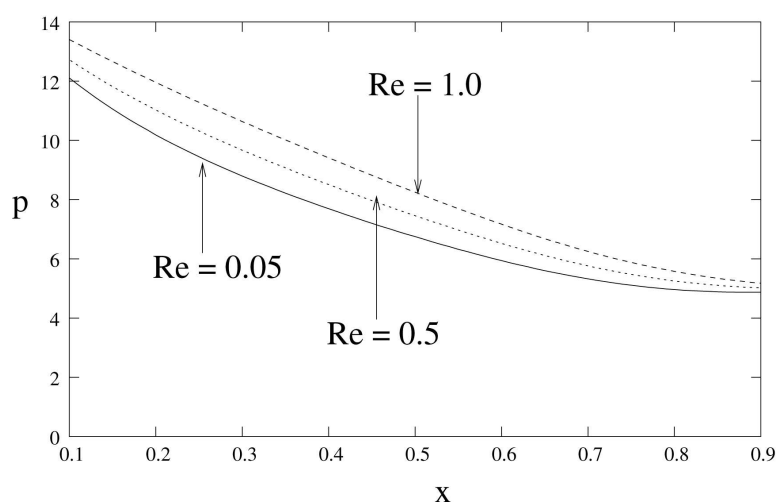

(a) Distribution of pressure $(p)$ with $x$. $(k=0.1, \alpha=0.5$, $\xi=0.15$.

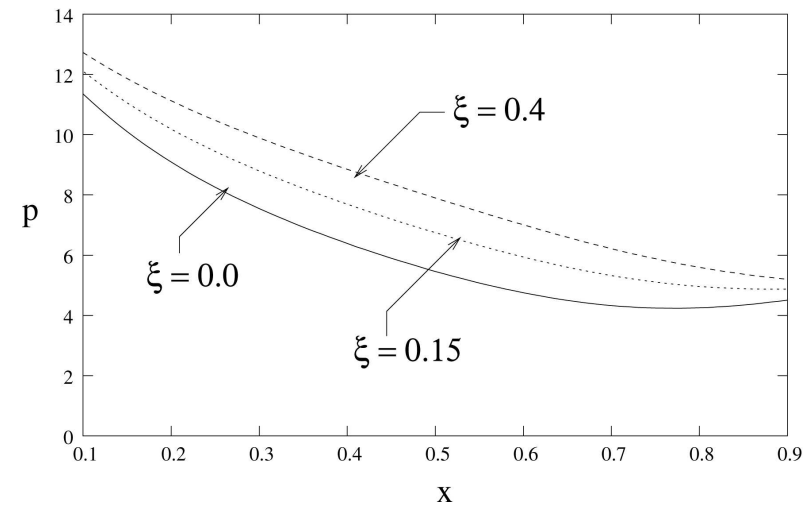

(b) Distribution of pressure $(p)$ with $x$. $(k=0.1, \alpha=0.5$, $R_{e}=0.05$.

Fig. 3

\subsection{The velocity $v$}

In this section, we discuss the effects of the slope parameter $(k)$, slip coefficient $(\xi)$ and permeability parameter $(\alpha)$ on the radial velocity. Also, we look into the behavior of the velocity at different cross sections of the tube. 
The effect of slope parameter $(k)$ on the radial velocity is shown in Fig. 4(a). As $k$ decreases, that is, as the tube changes from diverging to normal then to converging tube, the radial velocity decreases. Because as the space for the movement become close the flow speed of the fluid decreases.

Fig. 4(b) shows the behavior of the velocity as the fluid passes through the tube at different locations of $x$. As the fluid passes from the entrance to exit, the radial velocity decreases. Naturally, since the outflow of the fluid decreases with $\mathrm{x}$, the radial velocity has a lesser value at the exit than the entrance. Moreover, it attains the maximum at the point $\cong 0.6$ (when $\alpha$ is small) at the entrance and it shifts towards the boundary at the exit.

Fig. 4(c) shows the influence of slip coefficient $(\xi)$ on radial velocity. It is of interest to note that the effect of slip coefficient is to increase the radial velocity at the boundary. The effect of permeability parameter $\alpha$ is presented in the Fig. 5(a-c). It can be observed from these figures that as $\alpha$ increases, the radial velocity of the flow increases for all cases of $k$ ( converging, normal, and diverging tubes).

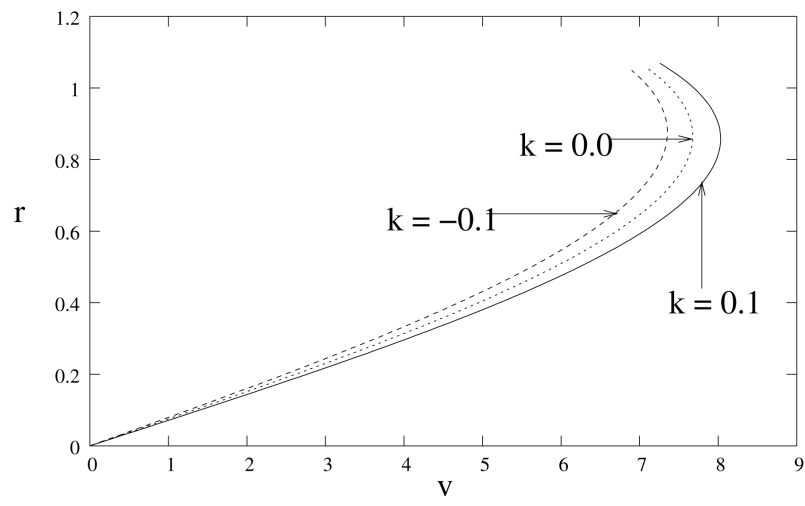

(a) Distribution of radial velocity $(v)$ with $r$. $\left(R_{e}=0.05, \alpha=\right.$ $0.5, x=0.1, \xi=0.15$.)

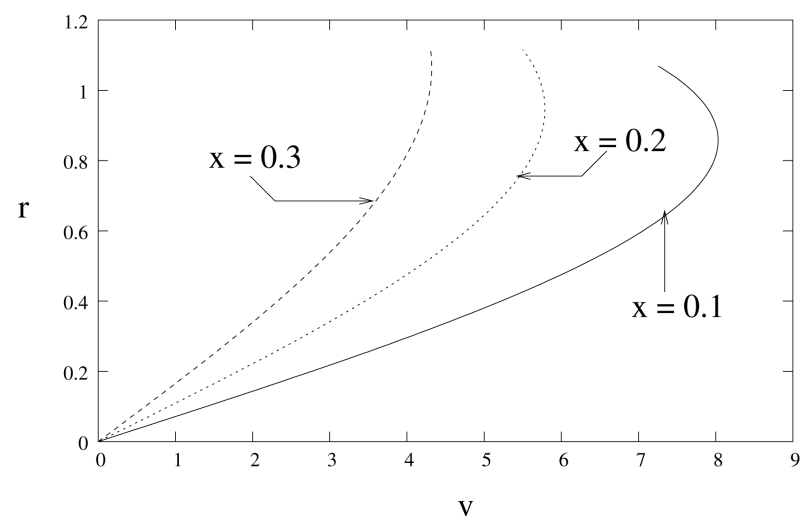

(b) Distribution of radial velocity $(v)$ with $r$. $\left(R_{e}=0.05, \alpha=\right.$ $0.5, \xi=0.15, k=0.1$. )

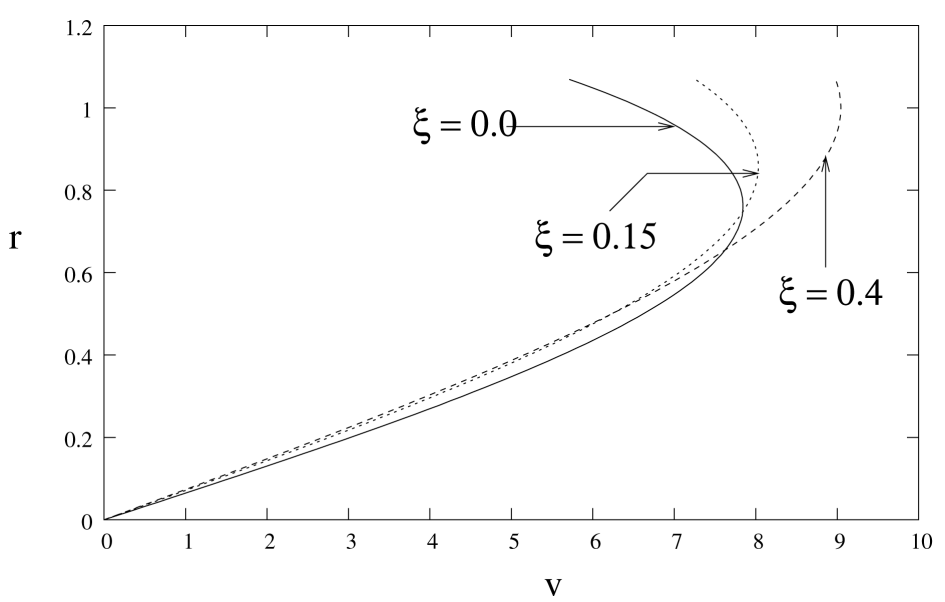

(c) Distribution of radial velocity $(v)$ with $r$. ( $R_{e}=0.05, \alpha=0.5, x=$ $0.1, k=0.1$.)

Fig. 4 


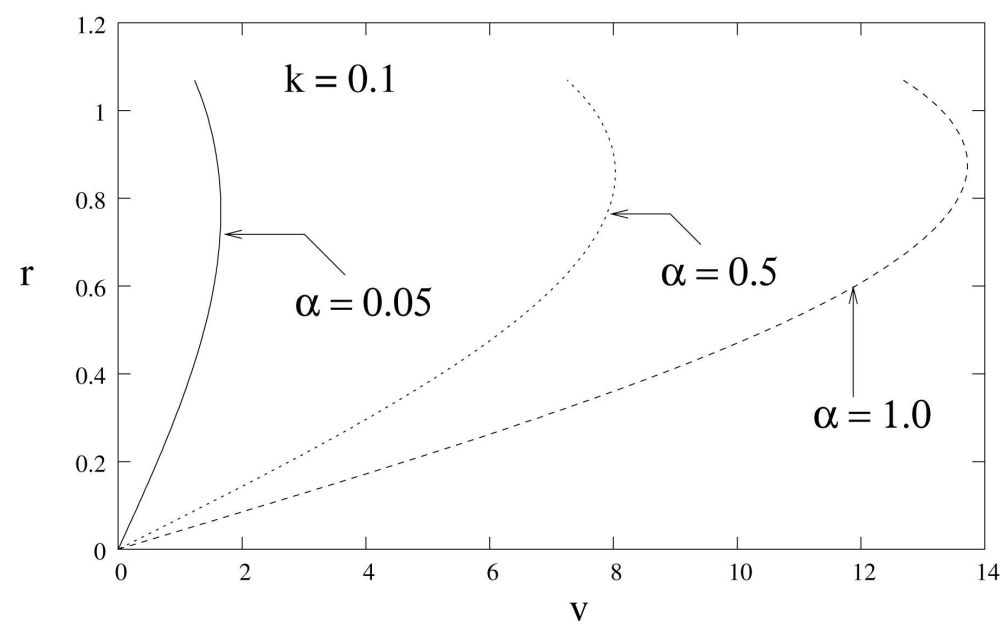

(a) Distribution of radial velocity $(v)$ with $r$. $\left(R_{e}=0.05, k=0.1, x=0.1\right)$.

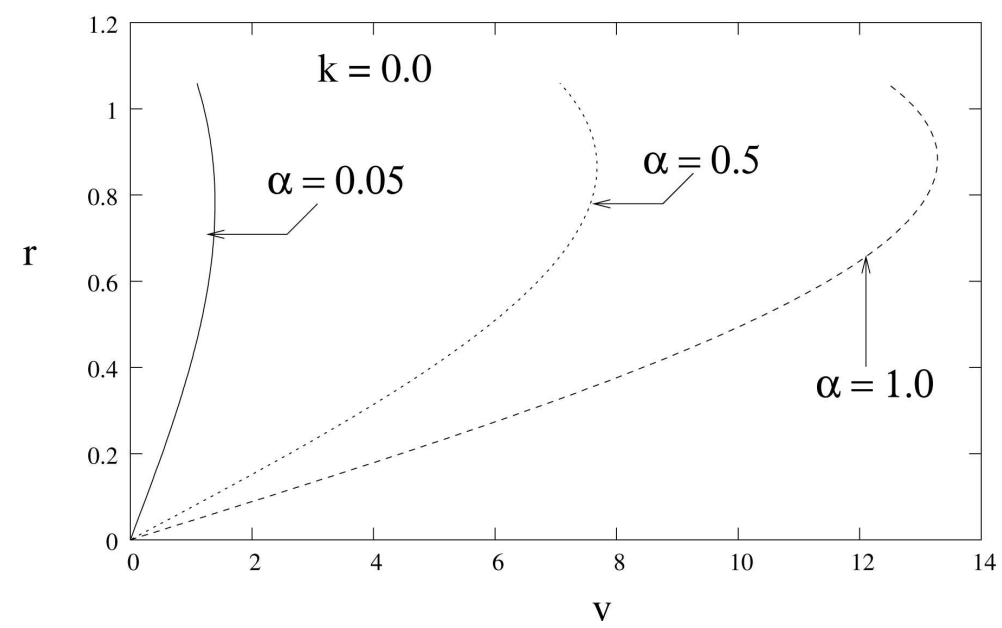

(b) Distribution of radial velocity $(v)$ with $r$. $\left(R_{e}=0.05, k=0.0, x=0.1\right)$.

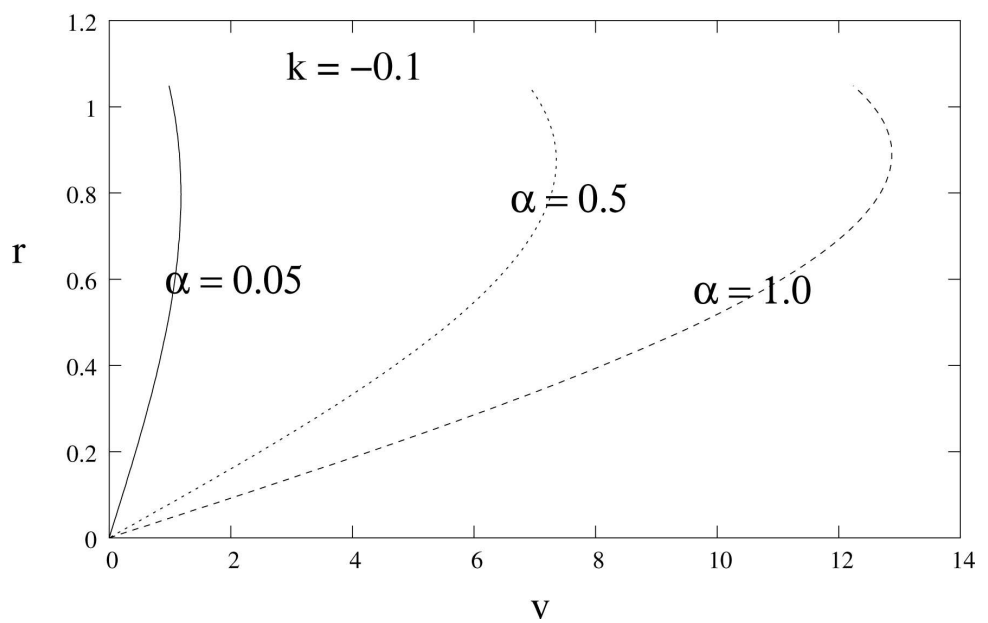

(c) Distribution of radial velocity $(v)$ with $r$. ( $\left.R_{e}=0.05, k=-0.1, x=0.1\right)$.

Fig. 5 


\subsection{Flow rate $Q$}

The effect of slope parameter $(k)$ on flow rate $Q$ is shown in Fig. 6(a). As $k$ increases, the flow rate increases. We observed that an increase in $k$ or the change of the tube from converging to diverging tube, increases the velocity which in turn results in more flow rate. Fig. 6(b) illustrates the effect of permeability parameter $(\alpha)$ on the flow rate $Q$ versus $x$. As shown, an increase in $\alpha$, produces an increase in flow rate $Q$ up to half of the tube and the reverse becomes true to the other half of the tube. This is because if the tube has high permeability, most of the fluid will reabsorbed at the entrance of the tube so that we will have a less flow rate at the exit.

Fig. 7(a) displays the influence of Reynolds number $R e$ on $Q$. The value of the flow rate $Q$ increases as $R e$ increases. Naturally, an increase in $R e$ increases the inertial effect which in turn results in more flow rate. Fig. 7(b) displays the influence of slip coefficient on $Q$. As shown, an increase in the slip coefficient $(\xi)$ increases the flow rate considerably.

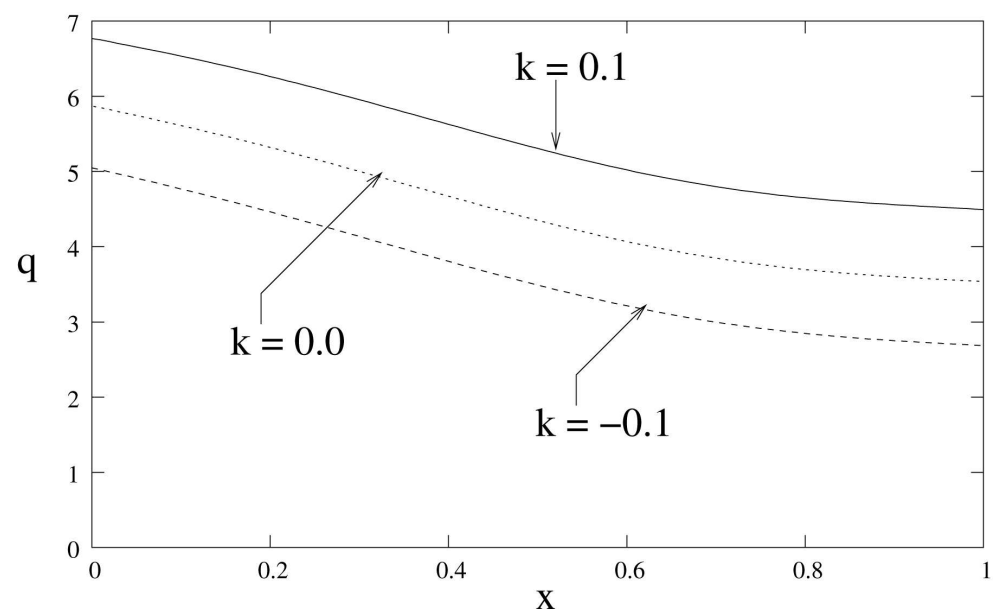

(a) Distribution of flow rate $(Q)$ with $x$. $\left(R_{e}=0.05\right.$,

$$
\alpha=0.05, \xi=0.15 \text { ). }
$$

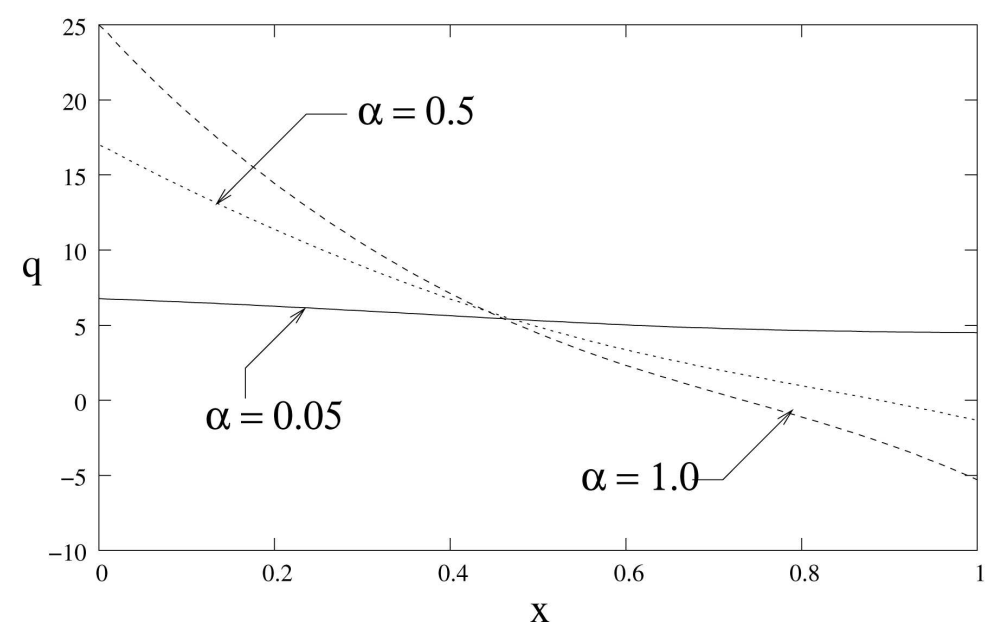

(b) Distribution of flow rate $(Q)$ with $x$. ( $R_{e}=0.05$,

$$
\xi=0.15, k=0.1) \text {. }
$$

Fig. 6 


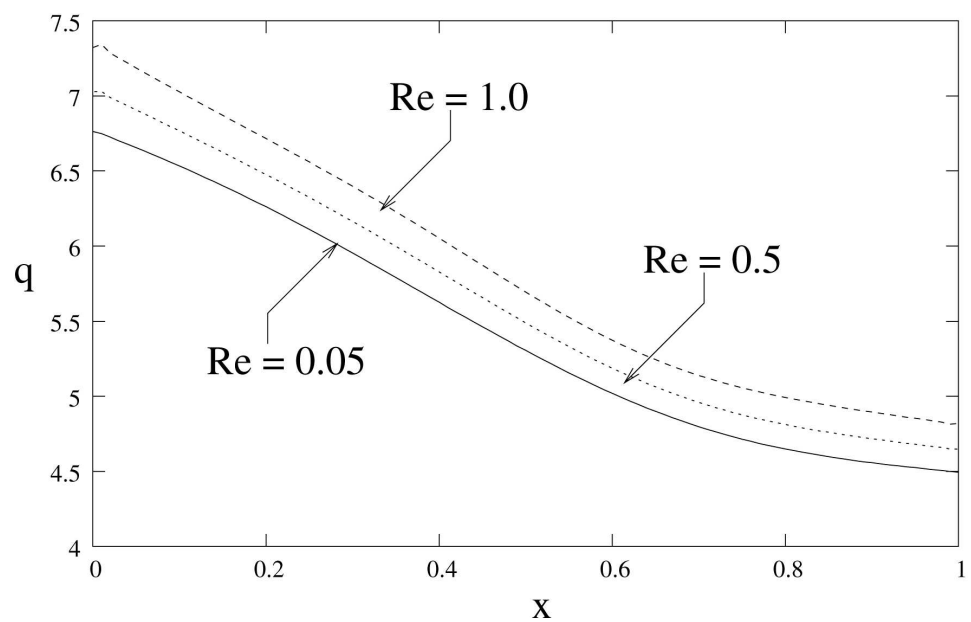

(a) Distribution of flow rate $(Q)$ with $x$. ( $k=0.1$, $\xi=0.15, \alpha=0.05)$

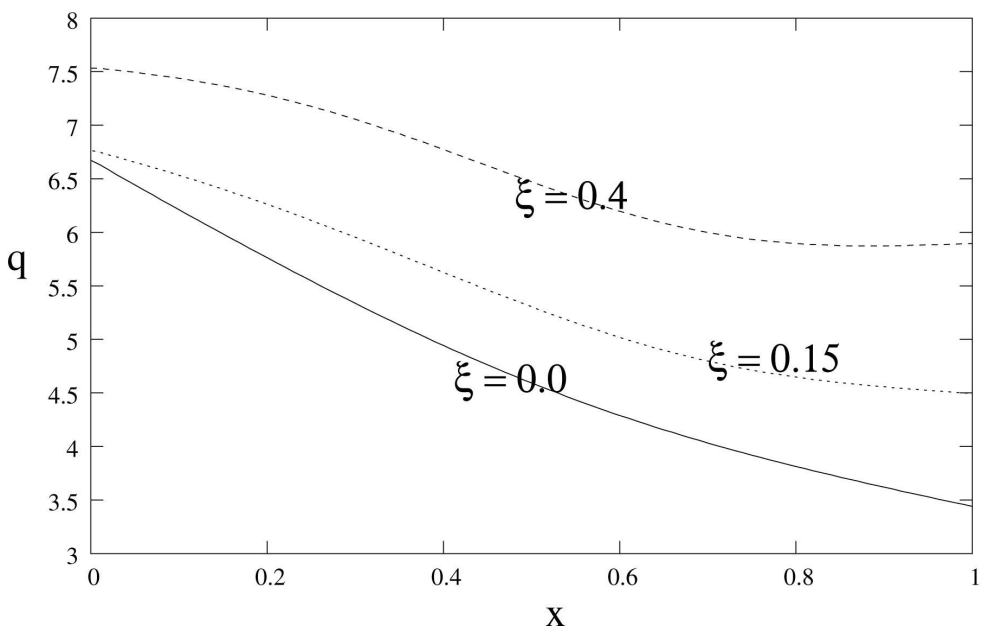

(b) Distribution of flow rate $(Q)$ with $x$. $\left(k=0.1, \alpha=0.05, R_{e}=0.05\right)$.

Fig. 7

\section{Conclusions}

In this study, a discussion of an incompressible fluid flow in a rigid tube of slowly varying converging/diverging wall has been presented with possible applications to the flow of fluid in renal tubules. The main contribution of this study is to show the effect of slip velocity at the boundary as it is not discussed in literature particularly to the renal flow. The reabsorption coefficient $\alpha$ and the slope parameter $k$ have the same effect on the radial velocity. As they increase, the velocity also increase. It is also of interest to note that the effect of slip coefficient $\xi$ is to increase the radial velocity at the boundary. When the permeability parameter $\alpha$ increases, the pressure decreases. This can justify the natural phenomenon that as most of the fluid goes out in a greater amount, the pressure is very less. The effect of slip coefficient $\xi$ is to increase the pressure and flow rate. $p$ is less for the divergent tube than the normal or convergent tubes, and it is more for convergent tube than the normal/divergent tubes. This is due to the fact that as the space for movement decreases, the velocity decreases which results in more pressure. 


\section{Competing interests}

The authors declare that they have no competing interests.

\section{Authors' contributions}

All authors have contributed to all parts of the article. All authors read and approved the final manuscript.

\section{References}

[1] R. B. Kelman. A Theoretical Note on Exponential Flow in the Proximal Part of the Mammalian Nephron. Bull. of Mathematical Biophysics, vol. 24, 303 - 317, 1962.

[2] Robert I. Macey. Pressure Flow Patterns in a Cylinder with Reabsorbing Walls . Bull. of Mathematical Biophysics, 25, 1 - 9, 1963.

[3] Robert I. Macey. Hydrodynamics in Renal Tubules. Bull. of Mathematical Biophysics, 27, 117-124, 1965.

[4] E. A. Marshall, and E. A. Trowbridge. Flow of a Newtonian Fluid through a Permeable Tube: The Application to the Proximal Renal Tubule. Bull. of Mathematical Biology, vol. 36, 457 - 476, 1974.

[5] Paul J. Palatt, Henry Sackin, and Roger I. Tanner. A Hydrodynamical Model of a Permeable Tubule. J. theor. Biol., 44, 287-303, 1974.

[6] G. Radhakrisnamacharya, Peeyush Chandra, and M. R. Kaimal. A Hydrodynamical Study of the Flow in Renal Tubules. Bull. of Mathematical Biology, vol. 43, No.2:151-163, 1981.

[7] Peeyush Chandra, and J.S.V.R. Krishna Prasad. Low reynolds number flow in tubes of varying cross-section with absorbing walls. Jour. Math. Phy. Sci., vol. 26, No.1, February 1992.

[8] P. Chaturani, and T. R. Ranganatha. Flow of Newtonian fluid in non-uniform tubes with variable wall permeability with application to flow in renal tubules. Acta Mechanica, 88, 11-26, 1991.

[9] G. S. Beavers, D. D. Joseph. Boundary conditions at a naturally permeable wall. Journal of Fluid Mechanics, 30, 197-207, 1967.

[10] P. Dulal, N. Rudraiah, D. Rathna. The effects of slip velocity velocity at a membrane surface on blood flow in the microcirculation. Journal of Mathematical Biology, 26, 705-712, 1988.

[11] I. J. Rao, T. Rajagopal. The effect of the slip boundary condition on the flow of fluids in channel. Acta Mechanica, 135, 113-126, 1999.

[12] Moustafa Elshahed. Blood flow in capillary under starling hypothesis. Applied mathematics and computation, 149, 431-439, 2004.

[13] P. Muthu and Tesfahun Berhane. Mathematical model of flow in renal tubules, Int. J. of Appl. Math. and Mech., 6(20), 94-107, 2010.

[14] Syoten Oka and Tadayoshi Murata, A Theoretical Study of the Flow of Blood in a Capillary with Permeable wall, Japanese Journal of Applied Physics, 9(4), p: 345-352, 1974.

[15] N. K. Mariamma and S. N. Majhi, Flow of a Newtonian Fluid in a Blood Vessel with Permeable Wall - A Theoretical Model, Computers and Mathematics with Applications, 40, p: 1419-1432, 2000. 